\section{PS-372 DIFFUSE FATTY INFILTRATION IN STRESS-RELATED THYMIC INVOLUTION: AN INDEPENDENT FINDING FOR PAEDIATRIC INFECTION}

${ }^{1} \mathrm{M}$ Taweevisit, ${ }^{2} \mathrm{P}$ Thorner. ${ }^{1}$ Pathology, Chulalongkorn University, Bangkok, Thailand; 2 Pathology and Laboratory Medicine, University of Toronto, Toronto, Canada

10.1136/archdischild-2014-307384.670

Background and aims Fatty infiltration is known as a physiologic hallmark of thymic involution, starting at puberty (agerelated involution). However, the knowledge of fatty infiltration in thymuses whose paediatric patients suffering from acute illness (stress-related involution) has never been studied. The purpose of this work was to evaluate the frequency and degree of fatty infiltration and to correlate the degree of fatty infiltration with the severity of involution in thymic tissues belonging paediatric patients who died from various causes of illness.

Methods Thymic tissues from paediatric autopsy series were collected and processed for histopathologic examination. The degree of fatty infiltration was divided semi-quantitatively as absence, minimal (<5\%), occasional (5-50\%) and diffuse $(>50 \%)$. The severity of thymic involution was graded as 1 (resting state), 2 (more thymic lobule separation) and 3 (advanced stromal fibrosis).

Results Fatty infiltration (Figure 1) was found 36/130 cases $(28 \%)$ and tended to accumulate in grade 3 thymic involution ( $p$ $=0.01)$. In most cases, the degree was minimal (11 cases; 9\%) or occasional (18 cases; $14 \%$ ) and no statistical correlation with any clinical information. There were 7 cases (5\%) showing diffuse fatty infiltration and all of them died from infection (bronchopneumonia $=3$, meningitis $=2$, acute myocarditis with abscesses $=1$, acute pyelonephritis $=1$ ), regardless of specific organism, patient age or duration of illness.

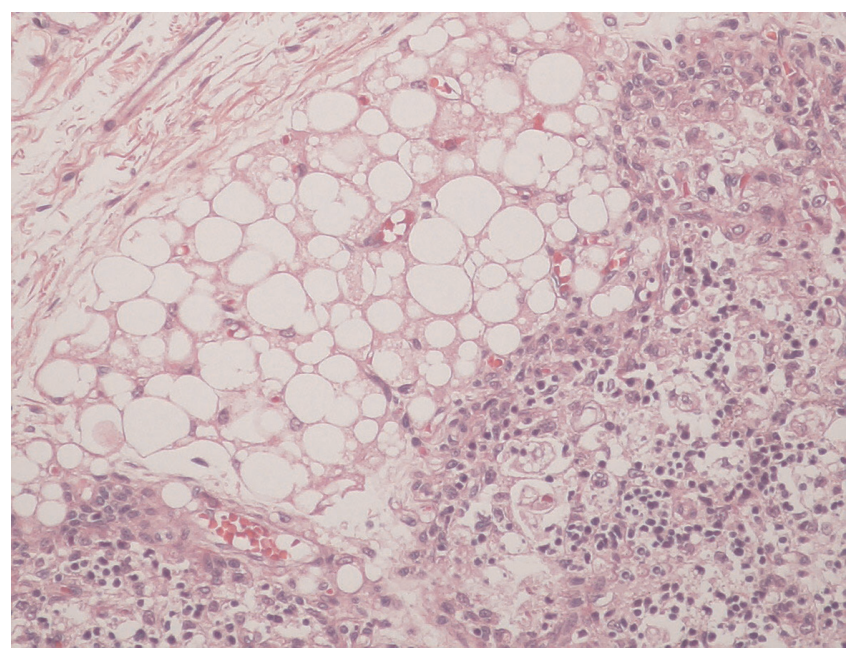

Abstract PS-372 Figure 1 Thymic fatty infiltration.

Conclusion In stress-related thymic involution, the frequency of diffuse fatty infiltration was rare, but such change was an independent finding associated with paediatric infection.

\section{PS-373 OPTIMISING A DIAGNOSTIC ALGORYTHM IN PATIENS PRESENTING FOR ADENOPATHY IN PRIMARY CARE SETTINGS}

R Bogdan, M Oros, C Codleanu, C Radu, O Dedu, A Dumineca, C Momirla. Pediatrics, Medicover Clinic, Bucharest, Romania
Aim To describe the etiologic spectrum, clinical characteristics and evaluate the adequateness of the tests performed in order to establish diagnosis of adenopathies in children.

Materials and method Our group analysed 279 children with adenopathies consulted in the primary care paediatric clinic in a 4 years period 2009-2013. There were noted: the presence and localization of the adenopathies, the diagnosis, the tests performed and the outcome.

Results Out of the 279 cases, 106 represented unspecific multiple adenopathies following past diseases and had no clinical significance. 77 cases were confirmed to be viral unspecific infections of the upper respiratory system, 16 cases were confirmed as Infectious mononucleosis, 22 cases were Acute bacterial Tonsilitis and 11 were confirmed as Group A Streptococcus Tonsilitis. Further investigation of cases with large adenopathies of unknown origin revealed less common etiologies as Toxocara and Toxoplasma infections. There was 1 case of Hodgkin lymphoma easily diagnosed based on clinical characteristics, confirmed and treated in the oncology hospital. 7 cases presented with typical left axilar satellite adenopathy following BCG vaccination. The history and clinical signs suggested the diagnosis in all the cases. In 27 of the cases with unique large adenopathies the parents refused further diagnostic tests.

Conclusions A complete history and a meticulous clinical examination represented the most important steps in establishing the etiologic diagnosis of the adenopathies in children. Specific blood tests and other investigations must be used with caution, in order to avoid unnecessary painful diagnostic procedures and unjustified costs.

\section{PS-374 A COMPARISON OF TREATMENT AT HOME OR IN HOSPITAL FOR MODERATE/SEVERE CELLULITIS IN CHILDREN}

${ }^{1}$ SM Hopper, ${ }^{2}$ LF Ibrahim, ${ }^{1} \mathrm{FE}$ Babl, ${ }^{3} \mathrm{PA}$ Bryant. 'Emergency Department, The Royal Children's Hospital and Murdoch Children's Research Institute, Parkville Melbourne, Australia; ${ }^{2}$ RCH@Home, The Royal Children's Hospital, Parkville Melbourne, Australia; ${ }^{3}$ RCH@Home Infectious Diseases Unit, The Royal Children's Hospital and Murdoch Children's Research Institute, Parkville Melbourne, Australia

\subsection{6/archdischild-2014-307384.672}

Background and aims Adults with cellulitis are commonly receive IV antibiotics via hospital-in-the-home (HITH). Children are usually admitted to hospital. Royal Children's Hospital (RCH) HITH and offers once daily IV ceftriaxone for cellulitis. Concerns remain for some physicians about its anti-staphylococcal activity. We aim to compare the clinical features and outcomes of patients with cellulitis admitted to hospital with IV flucloxacillin to those treated via HITH with IV ceftriaxone.

Methods A retrospective chart review of patients with cellulitis treated with IV antibiotics. Exclusions- complicated cellulitis (abscess, orbital cellulitis, post-operative cellulitis, bites and immunosuppression). Demographics, clinical and microbiological features, antibiotic management and outcomes are related to two groups: inpatients treated with IV flucloxacillin and HITH patients treated with IV ceftriaxone.

Results Over 17 months (2012-2014), 745 children presented to ED with cellulitis: 353 (47\%) received IV antibiotics; 169 were excluded (complicated cellulitis, comorbidities, misdiagnosis or miscoding), leaving 184. 47 (26\%) were admitted to HITH and 137 (74\%) were admitted as inpatients. Initial treatment was IV ceftriaxone in $41(87 \%)$ of HITH patients and IV flucloxacillin in 103 (75\%) of inpatients. 